\title{
Mechanical Vertical Manipulation of Selected Single Atoms by Soft Nanoindentation Using Near Contact Atomic Force Microscopy
}

\author{
Noriaki Oyabu, ${ }^{1}$ Óscar Custance, ${ }^{2, *}$ Insook Yi, ${ }^{2}$ Yasuhiro Sugawara, ${ }^{1,2}$ and Seizo Morita ${ }^{1,2}$ \\ ${ }^{1}$ Graduate School of Engineering, Osaka University, 2-1 Yamada-Oka, Suita, 565-0871, Japan \\ ${ }^{2}$ Handai Frontier Research Center (FRC), 2-1 Yamada-Oka, Suita, 565-0871, Japan
}

(Received 27 December 2002; published 2 May 2003)

\begin{abstract}
A near contact atomic force microscope operated at low-temperature is used for vertical manipulation of selected single atoms from the $\mathrm{Si}(111)-(7 \times 7)$ surface. The strong repulsive short-range chemical force interaction between the closest atoms of both tip apex and surface during a soft nanoindentation leads to the removal of a selected silicon atom from its equilibrium position at the surface without additional perturbation of the $(7 \times 7)$ unit cell. Deposition of a single atom on a created vacancy at the surface is achieved as well. These manipulation processes are purely mechanical, since neither bias voltage nor voltage pulse is applied between probe and sample. Differences in the mechanical response of the two nonequivalent adatoms of the $\operatorname{Si}(111)-(7 \times 7)$ with the load applied is also detected.
\end{abstract}

During the last few years, near contact atomic force microscope (NC-AFM) has shown a great potential for studding both conductive and nonconductive surfaces with atomic resolution [1]. At the present, true atomic resolution on metals [2,3], semiconductors [4,5], and nonconductive surfaces [6,7] is routinely obtained with $\mathrm{NC}$ AFM, which has also shown its potential as force spectroscopic tool [8], being sensitive to the chemical properties of the atomic species at the surface. Nevertheless, one of the most amazing capabilities of the scanning probe microscopy, the ability for manipulating surfaces at atomic scale, remains almost unexplored with NCAFM. In this field, the scanning tunneling microscope (STM) has widely shown to be an ideal tool for vertical and lateral manipulation of individual single atoms [9-12] and molecules [11,13-15], as well as bonds [16-18], opening fascinating new areas of research and approaching our ability for engineering up to the ultimate limits of fabrication [19]. This ability could be expanded to the nonconductive surfaces, in which it is difficult to operate STM, if one could manipulate single atoms with NC-AFM using only the mechanical interaction force acting between the tip apex and the surface. In this Letter we present, to our knowledge, the first experimental evidence of the reproducible mechanical vertical manipulation of selected single atoms of a surface by using NC-AFM. The method used for carrying out these experiments is based on a carefully controlled soft nanoindentation process. The results of vertical manipulation of $\mathrm{Si}$ adatoms of the $\mathrm{Si}(111)-(7 \times 7)$ surface are presented and a proposed manipulation mechanism is briefly commented.

The experiments were performed using a home-built low-temperature (LT) ultrahigh vacuum (UHV) NCAFM [20] with a base pressure of $5 \times 10^{-11}$ Torr. NCAFM was operated in the frequency modulation detection scheme [21], and constant excitation mode [22] was used for oscillating low resistivity commercial $n$-doped silicon cantilevers with a typical spring constant of $48 \mathrm{~N} / \mathrm{m}$ and first harmonic mechanical resonant frequency of $160 \mathrm{kHz}$. The measured quality factor in UHV at $78 \mathrm{~K}$ was 170000 . Before cooling down to LT and starting the experiments, both cantilever tip apex and sample surface were prepared in situ. Clean $\mathrm{Si}(111)-(7 \times 7)$ surface was obtained from a $n$-type As doped single crystal Si wafer by direct current heating, flashing the sample up to $1200^{\circ} \mathrm{C}$ with subsequent slowly cooling down from $900{ }^{\circ} \mathrm{C}$ to room temperature (RT). During sample preparation, vacuum pressure was kept below $1 \times 10^{-10}$ Torr. The Si tip apex was carefully cleaned up by argon-ion bombardment for $30 \mathrm{~min}$. with $0.6 \mathrm{keV}$ ion energy, $1 \times 10^{-6}$ Torr of partial $\mathrm{Ar}$ pressure, and normal incidence ion beam directly on the tip apex. The experiments were conducted at $78 \mathrm{~K}$ tip and sample temperature. During the experiments, both tip and sample were always electrically grounded.

For the mechanical vertical manipulation of selected single atoms, the following sequence of steps was applied. After taking an atomically resolved image over a region centered on one terrace of the surface, a specific atom of the imaged area was selected for being manipulated. The tip was positioned directly above it and a soft nanoindentation was applied. The nanoindentation was performed with the feedback loop disconnected ramping the sample towards the oscillating tip at a typical rate value of $0.3 \AA / \mathrm{sec}$ from the tip-sample distance at which the initial image was acquired. Since the cantilever was oscillating at its first resonant frequency, multiple nanoindentation processes were taking place during the sample approach, one per oscillation cycle. The nanoindentation process was carefully controlled by monitoring simultaneously both the variations of the cantilever oscillation amplitude and the cantilever frequency shift. 
Upon further indentation, a sudden jump in the frequency shift was normally detected. At this point, the ramp applied for moving the sample was stopped, the sample was retracted to the initial position, and the feedback was reactivated at the same frequency shift set point value used for the initial image acquisition. Finally, the surface was imaged again with the same parameters used for taking the initial image.

An example of the result of applying this experimental procedure to $\mathrm{Si}$ adatoms of the $\mathrm{Si}(111)-(7 \times 7)$ reconstruction is shown in Fig. 1. The selected Si corner adatom marked with a circle in Fig. 1(a) was removed from its initial position at the surface and a vacancy was created without additional perturbation of the $\mathrm{Si}(111)$ $(7 \times 7)$ unit cell [Fig. 1(b)]. This fact implies that at least three strong covalent bonds that fixed the $\mathrm{Si}$ adatom to the surface were broken during the nanoindentation. Successive mechanical manipulations can be performed over nearby adatom positions as shown in Fig. 1(c), where the corner adatom placed in the neighbor $(7 \times 7)$ unit cell, and marked with a circle in Fig. 1(b), was successively removed.

A soft nanoindentation can be also applied over a created vacancy, as the one shown in Fig. 2(b), generated by removing the central adatom marked with a circle in Fig. 2(a). The result of such an experiment was the deposition of a single atom that restored the initial unperturbed structure of the $\operatorname{Si}(111)-(7 \times 7)$ half-cell [Fig. 2(c)], that could infer the possible formation of three new covalent bonds since the deposited atom adapted to a central adatom position of the $\mathrm{Si}(111)$ $(7 \times 7)$ reconstruction.

A change in the contrast of the image acquired after performing a mechanical manipulation experiment can be sometimes observed. This effect is shown in the line profiles presented in Fig. 2. For the same image acquisition parameters, the line profiles taken at the same position in each of the three images show, in this case, an increase in the corrugation detected by the NC-AFM

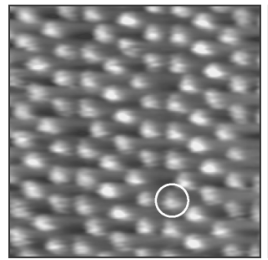

(a)

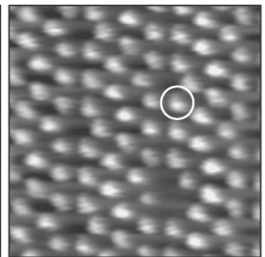

(b)

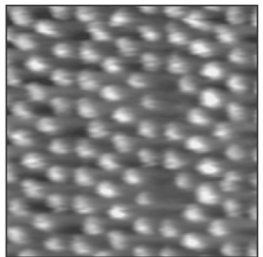

(c)
FIG. 1. Sequence of topographic images showing two mechanical single atom vertical manipulation processes performed successively over the selected atomic positions of the $\operatorname{Si}(111)-(7 \times 7)$ surface marked with a circle in (a) and (b), respectively, by using NC-AFM. As a result, two vacancies were created at the selected atomic positions (c). Image size is $66 \times 66 \AA^{2}$. Cantilever oscillation amplitude was $665 \AA$ and frequency shift reference value was $-1 \mathrm{~Hz}$ for all the images. after the removal and the deposition events, respectively. The change in the image contrast could be due to a modification of the atomic structure or a change of the dangling bond configuration of the tip apex, probably, by the interchange of atoms between tip and surface in the single atom mechanical manipulation event [23].

Previous STM works on manipulation of the bare $\operatorname{Si}(111)-(7 \times 7)$ surface pointed to a thermally activated field ion emission due to a high electric field between tip and sample during the application of a voltage pulse [24-26] as the mechanism for removing single Si adatoms at RT. This could be also the mechanism for the modification of the $\mathrm{Si}(111)-(7 \times 7)$ surface performed with LT NC-AFM by applying a voltage pulse between probe and sample recently reported by Sugawara et al. [27]. An alternative mechanism was proposed by Stipe et al. for the LT lateral displacement manipulation experiments of single Si adatoms performed by STM, where tunneling electrons from the STM tip occupying temporally a surface resonance state could play a fundamental role [16]. Nevertheless, the experiments presented in this work suggest that a completely different mechanism for manipulating $\mathrm{Si}$ adatoms is involved since neither bias voltage nor voltage pulse was applied between probe and sample during the manipulation process.

The selected single atom vertical manipulation experiments reported here could be explained as the result of a soft nanoindentation process. Figure 3 presents plots showing the typical evolution of the oscillation amplitude of the cantilever and the variation of the frequency shift

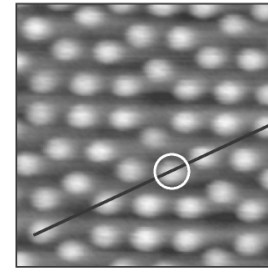

(a)

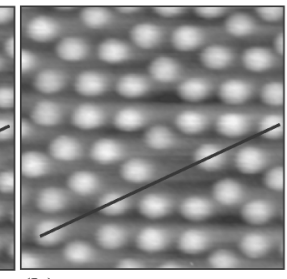

(b)

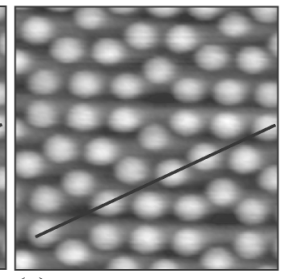

(c)

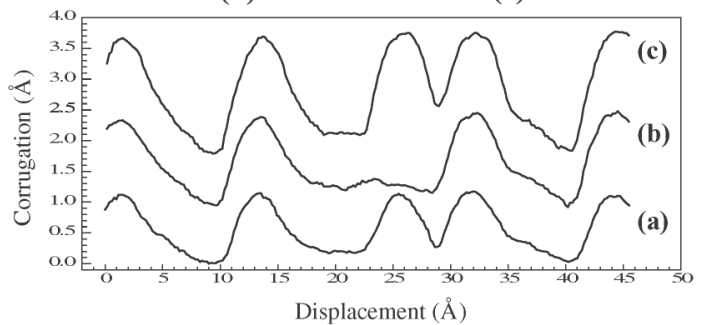

FIG. 2. Sequence of topographic images showing the deposition of a single atom (c) as a result of applying a soft nanoindentation process over a vacancy (b) previously created by removing the central adatom marked with a circle in (a). Line profiles taken along the diagonal of the $(7 \times 7)$ unit cell performed at the same position over each image are also shown. The line profiles are offset for clarity. Image size is $47 \times 47 \AA^{2}$. Cantilever oscillation amplitude and frequency shift reference values were $257 \AA$ and $-4 \mathrm{~Hz}$, respectively, for all the images. 


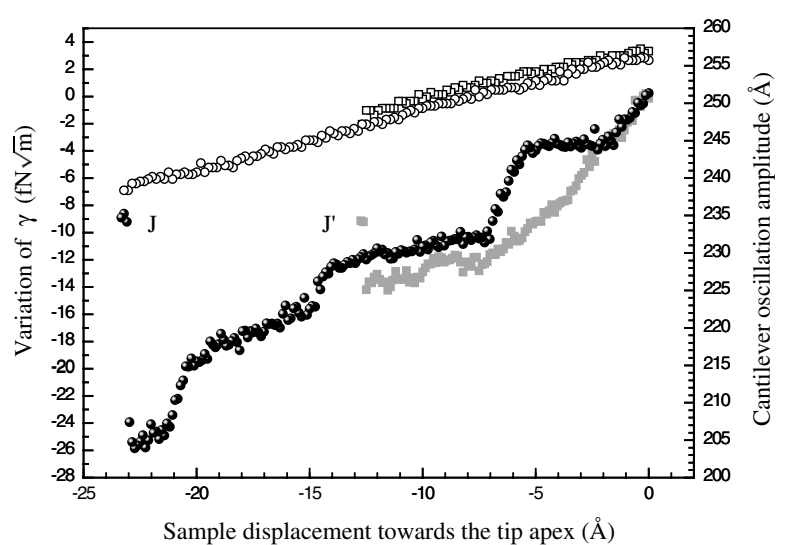

FIG. 3. Plot showing the evolution of the oscillation amplitude of the cantilever (open symbols) and the variation, respect to the initial value, of the normalized frequency shift $\gamma$ (solid symbols) when approaching the sample towards the tip during the removal process of a $\mathrm{Si}$ corner adatom (squares) and a $\mathrm{Si}$ central adatom (circles and spheres) of the Si(111)$(7 \times 7)$ reconstruction due to soft nanoindentation processes. Initial normalized frequency shift value for both curves was $-4.6 \mathrm{fN} \sqrt{m}$.

respect to the initial image value, using the normalized frequency shift $\gamma$ representation [28], during soft nanoindentation processes performed over a Si corner adatom and a Si central adatom, respectively, with the result of the adatom removal. In both cases, the increase in the energy loss of the oscillation cantilever by increasing the cyclic short-range chemical force interaction while reducing the tip-sample distance produces a decrease in the cantilever oscillation amplitude since the cantilever is oscillated in the constant excitation mode [22]. As approaching the sample towards the tip, before the indentation process starts, the frequency shift also decreases by increasing the attractive force interaction. Upon further approaching, the initial attractive chemical force that allows atomic resolution with NC-AFM [29] becomes strongly repulsive, starting the initial stages of the nanoindentation. The evolution of the frequency shift curves during the soft nanoindentation is characterized by several stages of different slope before the discontinuities chosen as the end of the process, labeled by $J$ and $J^{\prime}$ in Fig. 3, take place. Pérez et al. [30] have performed first principles simulations of a nanoindentation process in $\mathrm{Si}(111)$. They found that a nanoindentation performed over the two first atomic double layers of the surface is characterized by a pattern of successive elastic and plastic deformations. The plastic deformations, which seen as discontinuous jumps in the total energy of the system, were associated with breaking of few bonds, displacement of atoms, and remaking of bonds in a new stable configuration for releasing the stress accumulated during the elastic regime [30]. Although in those calculations the positions of the atoms at the tip were held fixed, it has been shown that in NC-AFM, atomic relaxation pro- cesses take place in both the tip apex and the surface for releasing the stress originated by the short-range chemical interaction forces in the attractive and repulsive regime [31]. Jumps in the total energy of the system associated with breaking of bonds manifest in visible discontinuities in a force versus tip-sample distance curve in NC-AFM as have been predicted by first principles calculations [32]. Hence, under a real experimental condition operating the NC-AFM in the frequency modulation scheme, these discontinuities should appear in a frequency shift versus tip-sample distance curve. The frequency shift curves shown in Fig. 3 reflect the elastic and plastic deformations that take place in the tip-surface system during the soft nanoindentation process. The discontinuity (jump) at the end of the curve ( $J$ and $\left.J^{\prime}\right)$ shows an important relaxation of the forces in the system, that could correspond to the plastic deformation associated with the breaking of bonds that allows a single adatom extraction, as was theoretically predicted [30]. Our data also indicate that, in the case of $\operatorname{Si}(111)-(7 \times 7)$ surface, the possible damage originated in layers below the adatom layer is very local and gentle since no perturbation of the neigboring adatoms of the half unit cell is observed after a soft nanoindentation and atoms can be deposited on created vacancies restoring the initial structure of this reconstruction as shown in Fig. 2.

Different shapes in the frequency shift curves associated to soft nanoindentation processes with the result of mechanical manipulation of single atoms have been detected during the experiments. This fact points out the possible relevance of the tip apex structure, composition, and orientation, since the structure of the $\operatorname{Si}(111)-(7 \times 7)$ surface at equivalent positions is expected to be the same. Although a wide spectrum of shapes has been obtained, the main characteristics of these curves are represented by the ones shown in Fig. 3. A detailed study of the tipsample system during a soft nanoindentation related with NC-AFM will be published elsewhere [33].

Figure 3 also points out the different response of the two nonequivalent $\mathrm{Si}$ adatoms of the $\mathrm{Si}(111)-(7 \times 7)$ reconstruction to the load applied over them. Although both frequency shift curves show the same tendency in the initial stages of the sample approach previous indentation process starts, the evolution is quite different. While the central adatom frequency shift curve shows alternating change between plateaus like stages and steeper slope stages over a relatively large tip-sample approach distance, the corner adatom frequency shift curve reaches the sudden discontinuity ( $J^{\prime}$ in Fig. 3 ) in shorter distance. On the contrary, almost no difference is detected in the behavior of the damping in the oscillation amplitude of the cantilever with the $\mathrm{Si}$ adatom type probed. Data presented in Fig. 3 indicate that the force applied for indenting and removing the central adatom is higher than the force applied in the case of the corner adatom. The experiments performed by Stipe et al. [16] suggest 
that the bonding energy of the corner adatom should be greater than the central adatom one, since they did not observe displacement of the corner adatoms in their LT STM single adatom manipulation experiments. Assuming this difference in the bonding energy and taken into account the different behavior of the nonequivalent adatoms under a soft nanoindentation process, data presented point out that the corner adatom covalent bonds and local structure seem to be stiffer than the central adatom ones since the sudden instability appears, in average, at shorter tip-sample displacements than in the central adatom case, indicating that less stress is relaxed when loading the corner adatom.

In this Letter it has been shown that NC-AFM allows enough precision for performing controlled soft nanoindentations involving the outermost atomic layers of the tip-surface system, that can be used for obtaining information of the local mechanical properties of the atomic bonds and local stress of a surface at atomic level. As a result of these carefully controlled soft nanoindentation processes, a method for vertical manipulation of selected single atoms with NC-AFM is proposed. This method is based only on short-range chemical interaction forces since neither bias voltage nor voltage pulse is applied between probe and sample. Although the work was done over the $\mathrm{Si}(111)-(7 \times 7)$ surface, this method could set the bases for performing single atom manipulation in nonconductive materials and structures, which are of great interest in many technological applications. However, both theoretical and experimental work have to be done for a full understanding of the soft nanoindentation process with NC-AFM and for obtaining quantitative measures of local hardness of the surface at atomic level.

This work was supported by the Handai Frontier Research Center and by a Grant in Aid for Science Research from the Ministry of Education, Culture, Sports, Science and Technology of Japan.

*Corresponding author.

Email address: oscar@ele.eng.osaka-u.ac.jp

[1] S. Morita, R. Wiesendanger, and E. Meyer, Noncontact Atomic Force Microscopy (Springer-Verlag, Berlin, 2002).

[2] S. Orisaka, T. Minobe, T. Uchihashi, Y. Sugawara, and S. Morita, Appl. Surf. Sci. 140, 243 (1999).

[3] Ch. Loppacher et al., Phys. Rev. B 62, 16944 (2000).

[4] F. J. Giessibl, Science 267, 68 (1995).

[5] Y. Sugawara, M. Ohta, H. Ueyama, and S. Morita, Science 270, 1646 (1995).
[6] M. Reichling and C. Barth, Phys. Rev. Lett. 83, 768 (1999).

[7] C. Barth and M. Reichling, Nature (London) 414, 54 (2001).

[8] M. A. Lantz et al., Science 291, 2580 (2001).

[9] D. M. Eigler and E. K. Schweizer, Nature (London) 344, 524 (1990).

[10] D. M. Eigler, C. P. Lutz, and W. E. Rudge, Nature (London) 352, 600 (1991).

[11] L. Bartels, G. Meyer, and K. -H. Rieder, Phys. Rev. Lett. 79, 697 (1997).

[12] G. Dujardin et al., Phys. Rev. Lett. 80, 3085 (1998).

[13] G. Meyer, B. Neu, and K. -H. Rieder, Appl. Phys. A 60, 343 (1995).

[14] T. A. Jung, R. R. Schlittler, J. K. Gimzewski, H. Tang, and C. Joachim, Science 271, 181 (1996).

[15] B. C. Stipe, M. A. Rezaei, and W. Ho, Science 279, 1907 (1998).

[16] B. C. Stipe, M. A. Rezaei, and W. Ho, Phys. Rev. Lett. 79, 4397 (1997).

[17] H. J. Lee and W. Ho, Science 286, 1719 (1999).

[18] J. R. Hahn and W. Ho, Phys. Rev. Lett. 87, 166102 (2001).

[19] A. J. Heinrich, C. P. Lutz, J. A. Gupta, and D. M. Eigler, Science 298, 1381 (2002).

[20] N. Suehira, Y. Tomiyoshi, Y. Sugawara, and S. Morita, Rev. Sci. Instrum. 72, 2971 (2001).

[21] T. R. Albrecht, P. Grütter, D. Horne, and D. Rugar, J. Appl. Phys. 69, 668 (1991).

[22] H. Ueyama, Y. Sugawara, and S. Morita, Appl. Phys. A 66, S295 (1998).

[23] As a result of a soft nanoindentation performed by using $\mathrm{NC}$-AFM, the removal or deposition of single atoms was normally achieved. Nevertheless, in some cases, two additional events could be observed after a soft nanoindentation. One was the deposition of material at the selected position in atom cluster form, presumably coming from the tip. The other was no detectable change observed in the surface even though a jump in the frequency shift was obtained.

[24] I. -W. Lyo and P. Avouris, Science 253, 173 (1991).

[25] A. Kobayashi, F. Grey, R. S. Williams, and M. Aono, Science 259, 1724 (1993).

[26] H. Uchida, D. Huang, F. Grey, and M. Aono, Phys. Rev. Lett. 70, 2040 (1993).

[27] Y. Sugawara, Y. Sano, N. Suehira, and S. Morita, Appl. Surf. Sci. 188, 285 (2002).

[28] F. J. Giessibl, Phys. Rev. B 56, 16010 (1997).

[29] R. Pérez, M. C. Payne, I. Štich, and K. Terakura, Phys. Rev. Lett. 78, 678 (1997).

[30] R. Pérez, M. C. Payne, and A. D. Simpson, Phys. Rev. Lett. 75, 4748 (1995).

[31] R. Pérez, I. Štich, M. C. Payne, and K. Terakura, Phys. Rev. B 58, 10835 (1998).

[32] S. H. Ke, T. Uda, R. Pérez, I. Štich, and K. Terakura, Phys. Rev. B 60, 11631 (1999).

[33] N. Oyabu et al. (to be published). 